


\section{Simultaneous Rocket and Satellite Measurements of the Topside Ionosphere}

\author{
S. J. Bayer, L. J. Blumle, J. L. Donlex, R. J. Fitzenreiter, and J \\ NASA・Goddard Space Flight Center, Greenbelt, Maryland
}

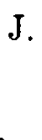

In view of the vast amount of data accumulating from Alouette, the Canadian topside sounder satellite [Chapman, 1963], it is bighly desirable to establish the accuracy of this technique by comparing the electron density profiles obtained by Alouette with similar data obtained independently. This accuracy affects the geophysical interpretation of the data as well as the possibility of using the topside sounder to calibrate ground-based techniques such as the incoherent backscatter radar. There are two possible sources of errors in the electron density profiles derived from topside sounders. The first one is associated with the method of converting the observed ionograms to true-height profiles. It has now been established that topside profiles agreeing within \pm 5 per cent in electron density (or within $\pm 15 \mathrm{~km}$ in altitude) are obtained by using various methods of analysis which differ significantly from each other in their underlying assumptions. A more serious problem is the uncertainty due to possible deviations of the propagation paths from the assumed vertical. Sometimes the presence of oblique propagation is quite obvious from ionograms whose analysis yields absurdly low values for the height of the $F_{2}$ maximum. In general, however, it may be quite difficult to assess the contribution of ray bending to the observed virtual depth and the resulting true height profile. Even matching topside and bottom soundings at the same location cannot quite resolve this uncertainty, because the height of the $F_{2}$ maximum (which is common to both observations) is the least accurate point determined by either sounding technique.

A much more satisfactory test of the accuracy of topside sounder measurements is to obtain a topeide profile at the same time and location by an independent and reliable technique. For this reason an ARGO D-4 rocket (NASA 8.14) was launched from Wallops Island, Virginia, July 2, 1963 , to intersect the Alouette orbit. This rocket carried two proven experiments, each yielding independently the charged-particle distribution in the topside ionosphere. The electron density was measured by the well-established twofrequency $\mathrm{CW}$ propagation technique as used in $186-$ previous high-altitude rocket flights [Jackson and $/ 89$ Bauer, 1961], in this case using 24.5- and 73.6$\mathrm{Mc} / \mathrm{s}$ signals. The ion density profile was measured by an ion retarding potential experiment employing a planar ion trap similar to that used in past rocket and satellite experiments [Donley, 1963; Bourdeau et al., 1962].

The date and time of the launching were selected to provide adequate proximity for a daytime comparison between the rocket and the satellite data. During the month of July 1963 these requirements could be met only on the 2nd and on the 14th, each day for a 10-minute period in the late morning. In addition, the practical requirements of the rocket flight, which yield a profile along the trajectory rather than vertically, dictated the need for stable and uniform ionospheric conditions in the area over which the comparison had to be made. The geometry and timing of this rendezvous experiment is shown in Figure 1. Although time simultaneity of the measurements could not be achieved for the entire rocket flight, it is seen in Figure 1 that the ascent data at altitudes between 300 and $600 \mathrm{~km}$ (corresponding to the altitude range in which the rocket data are most accurate) were obtained within minutes of the closest satellite data, and at an average horizontal distance of about $300 \mathrm{~km}$. The electron density profiles derived from the Alouette ionograms were virtually identical over the latitude range from $42^{\circ} \mathrm{N}$ to $37^{\circ} \mathrm{N}$, showing that there were no significant north-south gradients in the topside over a horizontal distance of at least $500 \mathrm{~km}$. In addition preflight monitoring of the ionospheric conditions using bottomside sounders at Wallops Island and at Ft. Belvoir had revealed uniformity in the east-west direc- 


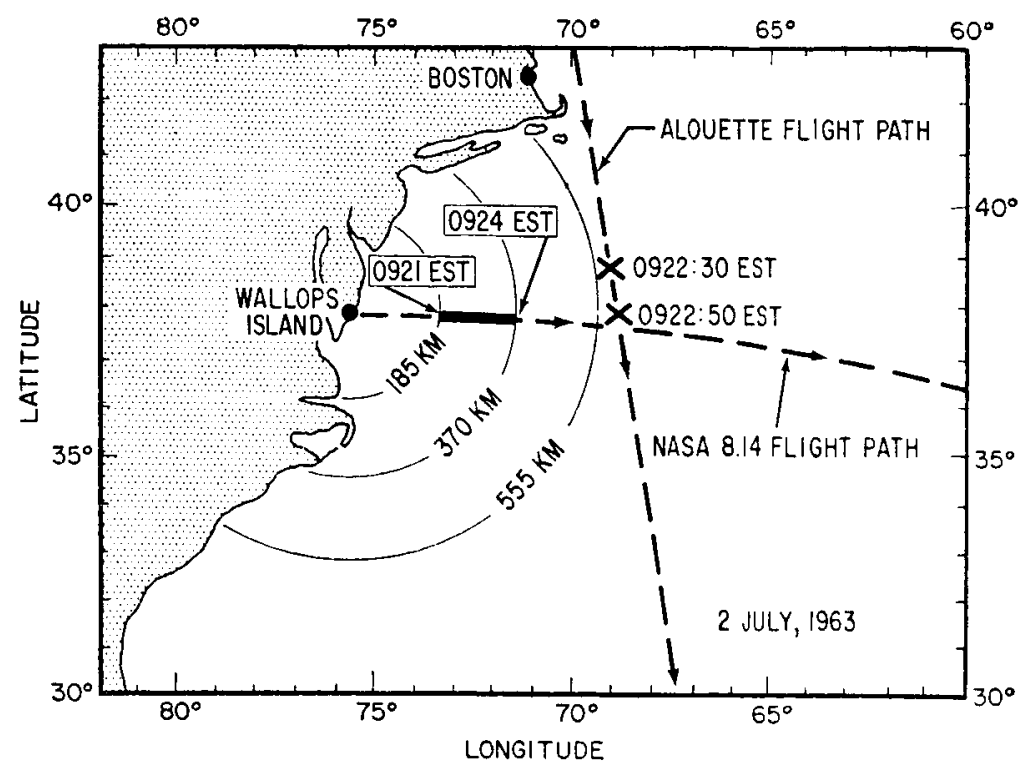

Fig. 1. Geometry of the experiment. The heavy part of the NASA 8.14 flight path indicates the horizontal range over which the ascent rocket data were for altitudes between 300 and $600 \mathrm{~km}$. Crosses on the Alouette flight path indicate locations and times corresponding to satellite soundings used for the comparison.

tion. Thus it appears that the requirement for a stable and uniform ionosphere was fairly well met during this test. This factor is also important, since another comparison was planned with an incoherent backscatter measurement made near Boston, Massachusetts $\left(42^{\circ} \mathrm{N}\right)$, by J. V. Evans.

The results of this test are given in Figure 2, which shows the charged-particle density profiles obtained by Alouette, the rocket-borne $\mathrm{CW}$ propagation and ion trap experiments, and the ground-based incoherent backscatter radar. The rocket data are most accurate for ascent and for altitudes below $700 \mathrm{~km}$, as the result of the trajectory achieved. Above $700 \mathrm{~km}$ the horizontal component of the rocket velocity became large compared to the vertical component, reducing the accuracy of the rocket data. For the CW propagation data, an obliquity correction has been made [Jackson and Bauer, 1961] which assumes that the ionosphere is stable and does not have significant horizontal gradients. Assuming an uncertainty of 10 per cent in this obliquity correction yields an electron density error of 5 per cent at $700 \mathrm{~km}$, increasing to about 15 per cent at $860 \mathrm{~km}$. Below $600 \mathrm{~km}$ the estimated error is less than 2 per cent.

The ion trap experiment yields ion densities from the measured collector current by assuming that the rocket velocity is larger than the ion thermal velocity and that the grid transparency for ions is the same as the optical transparency, which is consistent with results of previous flights [Donley, 1963]. The maximum error in ion densities is about 5 per cent (owing to calibration and telemetry scaling accuracy) below $700 \mathrm{~km}$ and about 10 per cent above $700 \mathrm{~km}$. The ion densities shown in Figure 2 include a correction which is required when the ion trap is not in the direction of the rocket velocity vector. In the upper part of the trajectory the angle of attack of the ion trap becomes larger, and this angle becomes an important factor in the analysis. The increased error in ion density above $700 \mathrm{~km}$ is due to the uncertainty in our knowledge of this angle of attack. No ion density data could be obtained below $367 \mathrm{~km}$ because of saturation of the ion trap.

The agreement between the two rocket-borne measurements is much better at altitudes above $700 \mathrm{~km}$ than could be expected on the basis of the stated uncertainties of either measurement. This agreement may be fortuitous, however, since comparison with the topside sounder at altitudes above $700 \mathrm{~km}$ reveals differences in density of at least 10 per cent, allowing an uncertainty due to analysis alone of about 5 


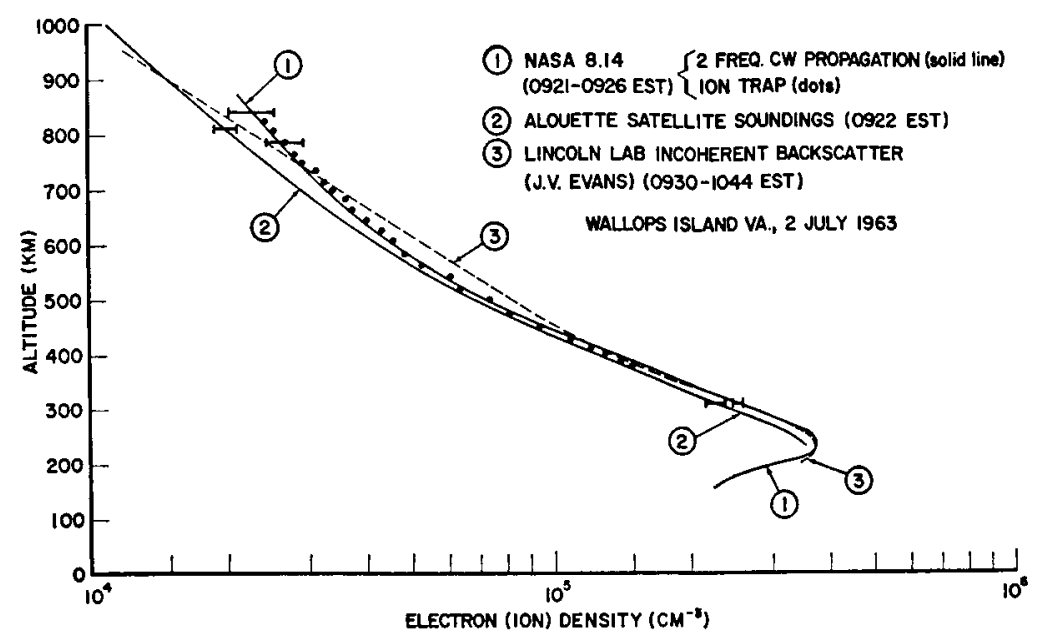

Fig. 2. Comparison of charged-particle profiles obtained by rocket, satellite, and groundbased incoherent backscatter measurements. Experimental errors in the rocket and satellite data are discussed in the text.

per cent in the topside sounder profile. A more significant comparison with the topside sounder data can be made below $600 \mathrm{~km}$, where the rocket data are believed to be correct within a few per cent; in this region the rocket and the satellite profiles agree within 8 per cent, which is only slightly greater than the combined uncertainty of the two measurements. Although $F_{2 \max }$ was poorly defined on the topside ionograms obtained on this occasion, the height of this peak inferred from the Alouette data appears to be too low by at least $20 \mathrm{~km}$, perhaps partly because of errors inherent in the true height analysis and partly because of propagation effect (slight ray bending).

A normalized electron density profile $\left(N / N_{\max }\right)$ derived from incoherent backscatter measurements, assuming that $T_{e} / T_{i}$, the ratio of electron to ion temperature is constant, was supplied by J. V. Evans. This normalized profile gave a height for the $F_{2}$ peak almost identical to that derived from the rocket data. The backscatter profile shown in Figure 2 was obtained by adjusting the normalized density profile at the $F_{2}$ peak to agree with the rocket data. There is excellent agreement between the adjusted backscatter data and the rocket data up to an altitude of about $450 \mathrm{~km}$, implying that $T_{0} / T_{i}$ may indeed be constant over this range. The disagreement at higher altitudes could be the result of a height dependence in the $T_{d} / T_{i}$ ratio; detailed information on this behavior must await the complete spectral analysis of the incoherent backscatter data.

Plotting the rocket data and the satellite data on a geopotential scale shows an essentially constant logarithmic slope from about 275 to 475 geopotential $\mathrm{km}$, indicating an almost constant scale height, $H^{\prime}=k\left(T_{0}+T_{i}\right) / m_{i} g_{0}$. Assuming that the mean ionic mass $m_{i}$ is 16 over this height interval, the upper limit of $\left(T_{a}+T_{i}\right)$ is $2500^{\circ} \pm 150^{\circ} \mathrm{K}$. Based upon the flux of solar decimeter radiation, the neutral gas temperature at the time of the test was estimated to be between $700^{\circ}$ and $800^{\circ} \mathrm{K}$. The effective charged-particle temperature $T_{i}+T_{i} / 2$ $=1250^{\circ} \mathrm{K}$ is thus significantly higher than the estimated gas temperature, indicating absence of thermal equilibrium over the specified altitude region. From the analysis of the retarding potential data obtained during the rocket flight, it may be possible to obtain also the variation of $T$. with altitude as well as some information concerning ion composition. When these data, as well as the spectral data from the incoherent backscatter become available, a more detailed interpretation of the charge density profile in terms of its structure parameters may become possible.

The above discussion illustrates some of the limitations of the topside sounder data and some of the difficulties encountered in determining the 
basic geophysical parameters from these profiles without additional information. From the standpoint of accuracy, however, it can be seen that the topside sounder profile compares well with those of other established techniques. In view of its broad geographic coverage, the topside sounder satellite appears to be the most useful tool at the present time for the synoptic study of the upper ionosphere, provided that the limitations of the technique are recognized and proper care is exercised in the analysis and interpretation of its data.

Acknowledgments. We are very grateful to Dr. J. V. Evans of the MIT Lincoln Laboratory for operating the incoherent backscatter radar during the Alouette test and for providing us with his preliminary results for this comparison.

Messrs. G. H. Spaid, R. J. Hagemeyer, G. A. Talley, and W. M. Daniels of the Goddard Space Flight Center were responsible for the design and execution of the CW propagation experiment. The personnel of the NASA Wallops Island Station deserve much credit for achieving the very precise timing of the rocket launch required for the experiment.

\section{REFERENCES}

Bourdeau, R. E., E. C. Whipple, J. L. Donley, and S. J. Bauer, Experimental evidence for the presence of helium ions based on Explorer 8 satellite data, J. Geophys. Res., 67, 467-475, 1962.

Chapman, J., Topside sounding of the ionosphere, Advan. Astronaut. Sci., 12, 43-63, 1963.

Donley, J. L., Experimental evidence for a low ion transition altitude in the upper nighttime ionosphere, J. Geophys. Res., 68, 2058-2060, 1963.

Jackson, J. E., and S. J. Bauer, Rocket measurement of a daytime electron density profile up to $620 \mathrm{~km}, J$. Geophys. Res., 66, 3055-3057, 1961.

(Received August 22, 1963; revised October 15, 1963.) 